\title{
Levantamiento topográfico de cuñas de diques forestales para estimar la erosión y emisión de sedimentos en cárcavas restauradas
}

\author{
Díaz, V. ${ }^{1}$ Ramos, I. ${ }^{2}$, Mongil, J. ${ }^{1}$ y Navarro, J..$^{*}$ \\ ${ }^{1}$ Grupo de Hidrología y Conservación de Aguas y Suelos. Universidad Católica de Ávila. \\ C/ Canteros, s/n 05005 Ávila \\ ${ }^{2}$ Dpto. de Ingeniería Agrícola y Forestal. Universidad de Valladolid. Avda. de Madrid, 34004 Palencia. \\ *e-mail: jnahev@iaf.uva.es
}

\section{Resumen}

La estimación de la erosión y emisión de sedimentos, empleando cuñas de diques, se viene estudiando en zonas de restauración hidrológico-forestal desde hace años. Para ello, suelen aproximarse los volúmenes de las cuñas a figuras geométricas sencillas. Como los resultados dependen del modelo geométrico empleado, en este trabajo se presenta una metodología basada en el levantamiento topográfico detallado $( \pm 1 \mathrm{~cm})$, de secciones transversales (espaciamiento 1-3 m) en la superficie de las cuñas. El método se aplicó en dos muestras de 25 y 15 diques, de badlands de Castilla y León restauradas durante el siglo pasado: Saldaña (Palencia), años 30, y Tórtoles (Ávila), años 60. Los resultados topográficos ofrecieron un volumen medio de sedimento por dique de $144 \mathrm{~m}^{3}$ (Saldaña) y de $17 \mathrm{~m}^{3}$ (Tórtoles), con valores totales entre un 4\% y un 24\% mayores, respectivamente, que con métodos geométricos. Consecuentemente, las tasas erosivas obtenidas con los métodos geométricos resultaron también inferiores. La tasa de sedimentos media retenida por dique desde su construcción, estimada topográficamente, fue entre $10.9 \mathrm{t}$ ha año ${ }^{-1}$ (Saldaña) y $6.4 \mathrm{t}$ ha año ${ }^{-1}$ (Tórtoles), mayor que las geométricas. La eficiencia de los diques según el método topográfico ha sido entre el 94 y el $98 \%$, mientras que con los geométricos entre el 90 y el 98\%. Dado que el levantamiento topográfico estima con mayor exactitud los sedimentos retenidos por los diques, se muestra como el método más apropiado para analizar el papel de estas obras en el control de sedimentos y para estimar la erosión a partir de sus cuñas.

Palabras clave: Control de erosión; control de sedimentos; gaviones; cárcavas; badlands. 


\section{Introducción}

Las áreas acarcavadas o badlands constituyen paisajes desertificados donde la vegetación es escasa o nula (Gallart et al., 2013), resultan inútiles para la agricultura (Bryan and Yair, 1982) y el tránsito de personas, vehículos y maquinaria es complicado. Estas áreas pueden aparecer sobre cualquier tipo de texturas y su génesis suele estar ligada a importantes desniveles geomorfológicos, cambios en la red de drenaje o procesos de tubificación (Mather et al., 2002). Generalmente están asociadas a altas pérdidas de suelo, inadmisibles en cuanto a su formación y conservación, así como a emisiones importantes de sedimentos y escorrentía durante los episodios de intensas precipitaciones (Clotet et al., 1987; García Ruiz et al., 2013; Nadal-Romero et al., 2013). Para frenar su avance y conseguir su restauración, los diques forestales junto con la reforestación juegan un papel fundamental.

Los diques forestales son estructuras bastante usuales en España (Martínez Azagra et al., 2002) empleadas, desde hace más de un siglo, en los proyectos de restauración hidrológico-forestal, por lo que resulta esencial conocer sus efectos y función en el control de sedimentos y de escorrentías (Castillo et al., 2007; Boix-Fayos et al., 2007; Romero et al., 2007; Díaz et al., 2014a). Estas hidrotecnias, que actúan como trampas de sedimentos, son importantes instrumentos para la determinación de tasas erosivas a nivel de cuenca (Romero et al., 2011), ya que el estudio de su registro sedimentológico, permite una interesante aproximación de la distribución espaciotemporal de la erosión y la producción total de sedimentos (Rodríguez-Lloveras et $a l ., 2014)$. Por ello, en diferentes ambientes mediterráneos españoles, se han empleado para determinar tasas erosivas y de emisión de sedimentos de las cuencas en que se emplazan (Castillo et al., 2007; Romero et al., 2007; Boix-Fayos et al., 2008; Sougnez et al., 2011; Bellín et al., 2011; Ramos et al., 2013; Díaz et al., 2014b). Los resultados obtenidos, según la metodología empleada, se aproximan en mayor o menor grado a la realidad sedimentaria (Ramos et al., en preparación).

Conviene señalar que no existe unanimidad entre los diferentes investigadores sobre la idoneidad de los diques empleados en las restauraciones hidrológico-forestales. Algunos plantean que generan más efectos perjudiciales aguas abajo del dique que beneficiosos en lo que a la fijación y conservación del suelo aguas arriba se refiere. Así se alerta que la construcción de obras hidráulicas puede alterar el régimen hidrológico del cauce y su morfología, generando impactos no deseados que anulen los efectos previstos en la redacción del proyecto (Boix-Fayos et al., 2007; Castillo et al., 2007, 2011). Estas afirmaciones se apoyan frecuentemente en el uso de modelos de estimación de la retención sencillos (Castillo et al., 2007; Romero et al., 2007; Bellín et al., 2011), propios para cauces tipo ramblas, pero que no son aplicables a otros cauces con sección en "V", más comunes en cárcavas.

Este estudio surge ante la necesidad de obtener con la mayor exactitud posible el volumen de las cuñas de sedimentos de los diques y, con una aproximación adecuada a la realidad, evaluar el papel de estas estructuras. De este modo, la realización de un levantamiento topográfico detallado de las cuñas mediante estación total, siguiendo el método de Díaz et al. (2014a, 2014b), en dos zonas acarcavadas de 
Castilla y León y la comparación de los resultados con otros modelos prismáticos frecuentes, permiten analizar la influencia de los métodos respecto a la obtención de tasas de erosión, emisión de sedimentos y la eficacia de retención de los diques forestales.

\section{Material y métodos}

\subsection{Zona de estudio}

El estudio se ha desarrollado en el área de Saldaña (Palencia) (UTM: 358400, 4707800; huso 30 N, Datum ETRS89) junto al río Carrión, y de Tórtoles (Ávila), próximo al río Corneja (UTM: 308563, 4492536; huso 30 N, Datum ETRS89), ambas en la cuenca del Duero (Fig. 1). Saldaña cuenta con una precipitación media anual de $528 \mathrm{~mm}$ y su temperatura media anual es $9.5^{\circ} \mathrm{C}$, mientras que en Tórtoles la precipitación media anual es $571 \mathrm{~mm}$, con una temperatura media anual de 10.6 ${ }^{\circ} \mathrm{C}$. Los materiales geológicos de Saldaña se corresponden con materiales Terciarios, transición entre los aluviones del Cuaternario del río Carrión y la cobertera arcilloarenosa y de acarreos cuarcíticos del Páramo del Terciario (IGME, 1982) que se extiende desde el pie de la Montaña Palentina. Tórtoles se encuentra sobre materiales plutónicos, tratándose en general de monzogranitos bióticos porfídicos de grano grueso (IGME, 2008). Los suelos, se corresponden con Entisoles del suborden Orthent para Saldaña y Orthents y Xerepts para Tórtoles (USDA, 2010). Además existe una marcada diferencia en cuanto a las texturas de los suelos, pasando de texturas fundamentalmente arcillosas en Saldaña a arenosas en la zona de Tórtoles. Estos

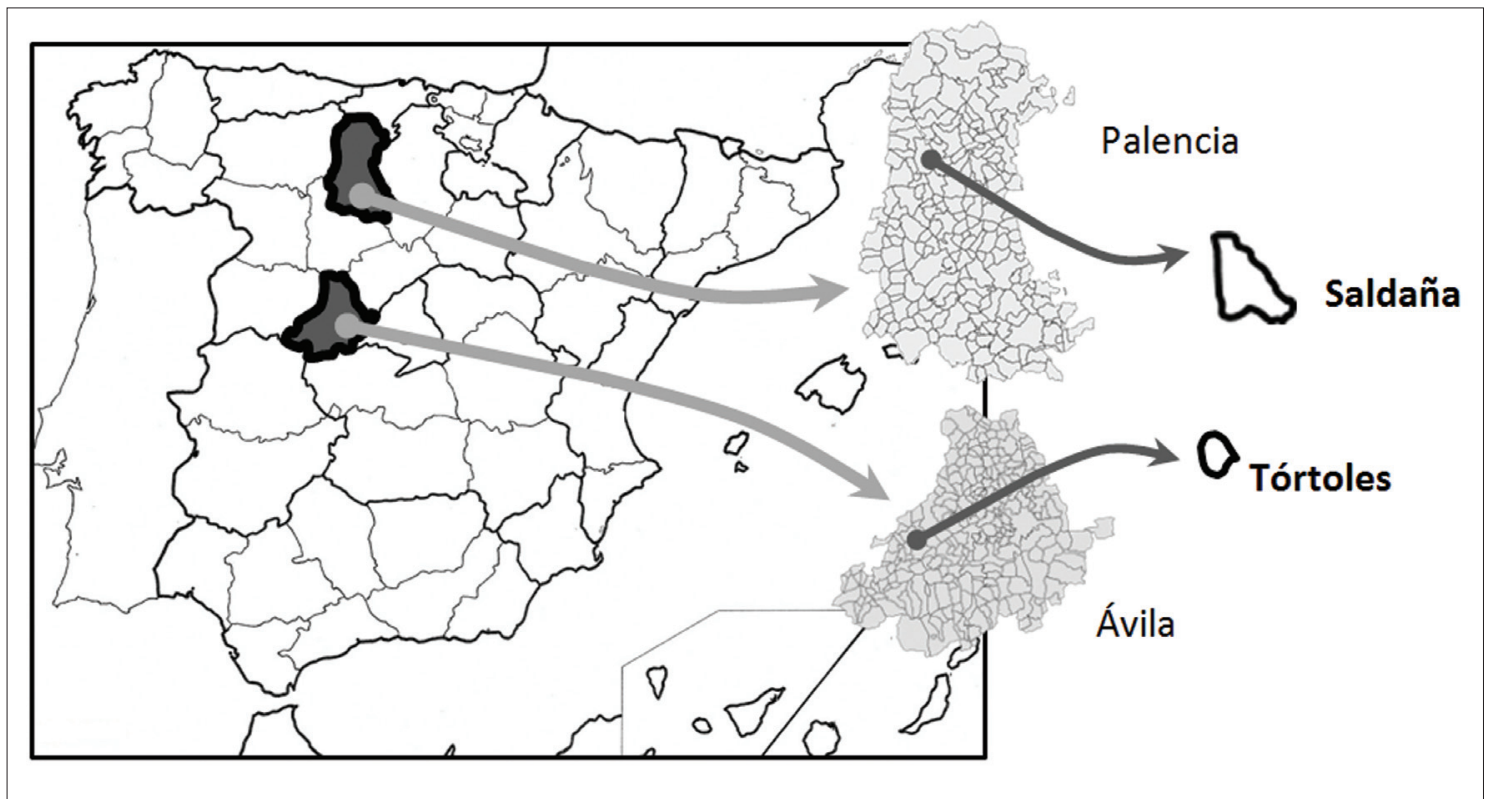

Figura 1. Localizacion de las zonas de estudio. 
lugares litológicamente diferenciados, fueron objeto de sendos proyectos de restauración hidrológico-forestal durante el siglo XX (Saldaña, en los años 30, por el ingeniero de montes D. José María Ayerbe; Tórtoles, en los años 60, por el ingeniero de montes D. David Azcarretazábal). Ambos proyectos plantearon la construcción de varios cientos de diques gavionados, así como la revegetación de las vertientes, para frenar los graves procesos erosivos que en ellos se venían desarrollando. En la actualidad, algo más de un centenar de diques existen en cada zona, donde los terrenos sustentan dos notables pinares que controlan la mayor parte de los procesos erosivos (Navarro et al., 2014; Mongil et al., en preparación).

\subsection{Estimación de la retención de sedimentos}

En primer lugar, se seleccionó la muestra de diques a medir para cada restauración (25 diques en Saldaña y 15 en Tórtoles) y se localizaron los diques objeto de estudio por medio de GPS. Estos se situaron mediante un punto en el centro del vertedero. Posteriormente, se procedió a determinar el volumen retenido por cada dique, mediante la medición de la cuña de sedimentos. Para ello, se aplicó el método topográfico propuesto por Díaz et al. (2014a, 2014b). Se trata de un levantamiento de precisión $( \pm 1 \mathrm{~cm})$, con estación total, de secciones transversales con equidistancia de 1 a $3 \mathrm{~m}$, en función de la longitud de la cuña de sedimentos y con una equidistancia de 0.5 a $1 \mathrm{~m}$ entre puntos dentro de cada sección transversal. Además, se levanta la sección aguas abajo del dique (con la misma equidistancia entre puntos) y la pendiente longitudinal del lecho del cauce. Los datos se analizan con AUTOCAD y se procesan ajustando las secciones transversales progresivamente, de acuerdo con la pendiente longitudinal y de las laderas, con la sección aguas abajo. Con estas secciones y mediante la hoja de calculo EXCEL, se determina el volumen (Vi) entre cada par de secciones consecutivas, aplicando la fórmula del prismoide (Ec. 1), que es la de mayor precisión en el cálculo de estos volúmenes (Díaz et al., 2014a).

$$
V_{i}=\frac{d_{i, i+1}}{6} \cdot\left(B_{i}+B_{i+1}+4 B_{m ; i, i+1}\right)
$$

Donde: $d_{\mathrm{i}, \mathrm{i}+1}$ es la distancia entre secciones "i" e " $\mathrm{i}+1$ ", a calcular; $B_{\mathrm{i}}$ y $B_{\mathrm{i}+1}$ son el área de las secciones extremas y $B_{\mathrm{m} ; \mathrm{i}, \mathrm{i}+1}$ es el área de la sección media.

Una vez obtenido el volumen de sedimentos retenido por dique $\left(\mathrm{V}=\sum_{\mathrm{i}=1}^{\mathrm{i}=\mathrm{n}} \mathrm{V}_{\mathrm{i}}\right)$, se calcula su peso teniendo en cuenta la densidad aparente del suelo, determinada con el método de los cilindros. En función del área vertiente hacia cada dique y el peso del sedimento retenido se pueden determinar los umbrales de producción de sedimentos para cada cuenca y dique analizado así como para el conjunto de las zonas.

Los resultados obtenidos se han comparado respecto a otros modelos prismáticos de uso frecuente como: el propuesto por Castillo et al. (2007), donde se plantea que los sedimentos retenidos siguen la forma de un canal trapezoidal determi- 
nado a partir de la sección media; y el formulado por Romero et al. (2007), en el cual lo asemejan a una pirámide de base trapezoidal en posición horizontal.

\subsection{Eficiencia de retención (TE) de los diques}

Los diques de gaviones permiten el paso del sedimento más fino a través de la obra debido a su permeabilidad. También se producen pérdidas de sedimento cuando circulan caudales por sus vertederos, ya que el tiempo de retención del flujo es menor que el de decantación de las arcillas. Por tanto, la eficiencia de retención (TE) del dique es normalmente inferior al 100\%. Para determinar TE existen diferentes métodos (Verstraeten and Poesen, 2000), aunque mayormente se ha obtenido mediante la expresión empírica propuesta por Brown and Jarvis (1943) (Ec. 2), como es el caso de los estudios de Bellín et al. (2011), Romero et al. (2012) y Díaz et al. (2014b).

$$
T E=100 \cdot\left(1-\frac{1}{1+0,0021 \cdot D \cdot \frac{C}{W}}\right)
$$

Donde: $C$ sería la capacidad de almacenamiento del dique $\left(\mathrm{m}^{3}\right) ; W$ el área de captación de sedimentos $\left(\mathrm{km}^{2}\right)$; D un parámetro empírico que adquiere valores entre 0.046 y 1 , con valor medio de 0.1 , dependiendo de las características del depósito. Brown and Jarvis (1943) sugieren que los valores para $D$ están cerca de 1 en aquellos casos donde el depósito se encuentra en una zona de baja y variable escorrentía, lo que se relaciona con una alta $T E$, como ocurre en nuestro caso.

\subsection{Producción (TSY) y emisión (SE) de sedimentos}

El sedimento retenido por los diques $(S Y)$, debido a la permeabilidad de estos, no se corresponde con la totalidad del sedimento producido en la cuenca (TSY). Para su obtención, primero se multiplica $S Y$ por el valor obtenido de $T E$ para cada dique. Este resultado ofrece la emisión de sedimentos $(S E)$, fracción del total de los sedimentos producidos en la zona de estudio que no han sido retenidos por los diques. Finalmente $T S Y$ se obtiene sumando $S Y$ y $S E$ (Ec. 3):

$$
T S Y=S Y+S E
$$

Donde: $T S Y$ es la producción total de sedimentos o tasa de erosión ( $\left.\mathrm{t} \mathrm{ha}^{-1} \mathrm{año}^{-1}\right)$; $S Y$ son los sedimentos retenidos o producción de sedimentos $\left(\mathrm{t} \mathrm{ha}^{-1} \mathrm{año}^{-1}\right)$ y $S E$ es la emisión de sedimentos ( $\mathrm{t}$ ha $\left.{ }^{-1} \mathrm{año}^{-1}\right)$.

\section{Resultados y discusión}

Los volúmenes de los diques calculados según el método topográfico de Díaz et 
al. (2014a, 2014b), del prisma trapezoidal (Castillo et al., 2007) y del prisma piramidal (Romero et al., 2007) se adjuntan en la Tab. 1 (Saldaña) y en la Tab. 2 (Tórtoles). Igualmente, en la $T a b .3$ y 4 se adjuntan $S Y, T E, S E$ y $T S Y$ para cada dique y para cada zona.

De los resultados obtenidos se desprende que el método topográfico ofrece un mayor volumen y peso de sedimentos que los otros dos métodos. Por ello, el método de Díaz et al. (2014a, 2014b) implica una mayor cantidad de sedimento retenido $\mathrm{y}$, por tanto, a partir de estos datos se obtienen unas tasas de erosión mayores en la zona (TSY), así como una mayor TE.

Tabla 1. Volúmenes (V), densidad y pesos (W) del sedimento retenido por los diques de Saldaña, según los métodos: topográfico (A), prisma trapezoidal (B) y prisma piramidal (C).

\begin{tabular}{|c|c|c|c|c|c|c|c|}
\hline $\mathbf{N}^{\circ}$ Dique & $\begin{array}{c}\mathbf{V}_{\mathbf{A}} \\
\left(\mathrm{m}^{3}\right)\end{array}$ & $\begin{array}{l}\mathbf{V}_{\mathbf{B}} \\
\left(\mathrm{m}^{3}\right)\end{array}$ & $\begin{array}{l}\mathbf{V}_{\mathbf{c}} \\
\left(\mathrm{m}^{3}\right)\end{array}$ & $\begin{array}{l}\text { Densidad } \\
\qquad\left(\mathrm{t} \mathrm{m}^{-3}\right)\end{array}$ & $\begin{array}{l}\mathbf{W}_{\mathrm{A}} \\
(\mathrm{t})\end{array}$ & $\begin{array}{l}\mathbf{W}_{\text {B }} \\
(\mathrm{t})\end{array}$ & $\begin{array}{l}\mathbf{W}_{\mathrm{C}} \\
(\mathrm{t})\end{array}$ \\
\hline 1 & 234.46 & 101.02 & 119.80 & 1.54 & 361.07 & 155.56 & 184.49 \\
\hline 2 & 610.72 & 754.87 & 769.54 & 1.52 & 928.29 & 1147.40 & 1169.70 \\
\hline 3 & 228.40 & 136.66 & 112.65 & 1.65 & 376.86 & 225.48 & 185.88 \\
\hline 4 & 386.97 & 316.43 & 262.46 & 1.7 & 657.84 & 537.93 & 446.19 \\
\hline 5 & 102.35 & 54.71 & 45.47 & 1.57 & 160.69 & 85.90 & 71.39 \\
\hline 6 & 109.48 & 74.87 & 59.07 & 1.65 & 180.64 & 123.53 & 97.47 \\
\hline 7 & 222.60 & 252.05 & 290.10 & 1.66 & 369.51 & 418.41 & 481.56 \\
\hline 8 & 121.86 & 101.98 & 92.06 & 1.66 & 202.28 & 169.29 & 152.81 \\
\hline 9 & 65.98 & 82.53 & 91.33 & 1.54 & 101.61 & 127.10 & 140.64 \\
\hline 10 & 115.11 & 103.52 & 95.58 & 1.58 & 181.87 & 163.56 & 151.02 \\
\hline 11 & 114.99 & 101.74 & 97.83 & 1.62 & 186.28 & 164.82 & 158.49 \\
\hline 12 & 93.26 & 115.74 & 103.44 & 1.53 & 142.69 & 177.08 & 158.26 \\
\hline 13 & 10.99 & 18.53 & 12.70 & 1.65 & 18.13 & 30.58 & 20.95 \\
\hline 14 & 44.29 & 77.86 & 66.98 & 1.77 & 78.39 & 137.82 & 118.55 \\
\hline 15 & 164.90 & 192.33 & 190.32 & 1.66 & 273.74 & 319.27 & 315.94 \\
\hline 16 & 66.95 & 97.05 & 85.57 & 1.56 & 104.44 & 151.40 & 133.48 \\
\hline 17 & 202.73 & 242.69 & 232.10 & 1.62 & 328.42 & 393.15 & 375.99 \\
\hline 18 & 6.14 & 8.53 & 9.65 & 1.76 & 10.81 & 15.01 & 16.99 \\
\hline 19 & 9.34 & 16.91 & 15.02 & 1.62 & 15.13 & 27.40 & 24.33 \\
\hline 20 & 7.67 & 14.659 & 18.59 & 1.63 & 12.50 & 23.89 & 30.30 \\
\hline 21 & 169.51 & 201.58 & 159.10 & 1.46 & 247.48 & 294.31 & 232.28 \\
\hline 22 & 177.02 & 148.14 & 125.86 & 1.38 & 244.28 & 204.44 & 173.69 \\
\hline 23 & 192.77 & 138.38 & 130.62 & 1.49 & 287.22 & 206.19 & 194.63 \\
\hline 24 & 79.38 & 51.33 & 60.41 & 1.49 & 118.28 & 76.48 & 90.01 \\
\hline 25 & 70.92 & 68.25 & 74.75 & 1.46 & 103.55 & 99.64 & 109.13 \\
\hline Total & 3608.74 & 3472.36 & 3320.97 & - & 5691.97 & 5521.06 & 5280.35 \\
\hline Media & 144.35 & 138.89 & 132.84 & 1.59 & 227.68 & 219.03 & 209.37 \\
\hline
\end{tabular}


Tabla 2. Volúmenes (V), densidad y pesos (W) del sedimento retenido por los diques de Tórtoles, según los métodos: topográfico (A), prisma trapezoidal (B) y prisma piramidal (C).

\begin{tabular}{|c|c|c|c|c|c|c|c|}
\hline $\mathbf{N}^{\circ}$ Dique & $\begin{array}{c}\mathbf{V}_{\mathbf{A}} \\
\left(\mathrm{m}^{3}\right)\end{array}$ & $\begin{array}{c}\mathbf{V}_{\mathbf{B}} \\
\left(\mathrm{m}^{3}\right)\end{array}$ & $\begin{array}{c}\mathbf{V}_{\mathbf{C}} \\
\left(\mathrm{m}^{3}\right)\end{array}$ & $\begin{array}{c}\text { Densidad } \\
\left(\mathrm{t} \mathrm{m}^{-3}\right)\end{array}$ & $\begin{array}{c}\mathbf{W}_{\mathbf{A}} \\
(\mathrm{t})\end{array}$ & $\begin{array}{c}\mathbf{W}_{\mathbf{B}} \\
(\mathrm{t})\end{array}$ & $\begin{array}{c}\mathbf{W}_{\mathbf{C}} \\
(\mathrm{t})\end{array}$ \\
\hline 1 & 7.95 & 6.53 & 6.00 & 1.30 & 10.32 & 8.47 & 7.79 \\
\hline 2 & 24.14 & 13.23 & 14.39 & 1.27 & 30.55 & 16.75 & 18.21 \\
\hline 3 & 7.58 & 6.17 & 6.78 & 1.28 & 9.67 & 7.88 & 8.65 \\
\hline 4 & 5.13 & 3.32 & 2.81 & 1.27 & 6.51 & 4.21 & 3.57 \\
\hline 5 & 34.08 & 28.48 & 25.95 & 1.34 & 45.64 & 38.14 & 34.76 \\
\hline 6 & 11.15 & 6.09 & 6.21 & 1.45 & 16.22 & 8.86 & 9.03 \\
\hline 7 & 24.82 & 26.86 & 22.36 & 0.98 & 24.29 & 26.30 & 21.89 \\
\hline 8 & 7.16 & 4.44 & 5.57 & 1.27 & 9.13 & 5.65 & 7.10 \\
\hline 9 & 50.49 & 32.33 & 34.11 & 1.25 & 63.02 & 40.35 & 42.57 \\
\hline 10 & 1.05 & 0.63 & 1.07 & 1.43 & 1.49 & 0.89 & 1.53 \\
\hline 11 & 44.64 & 47.77 & 54.86 & 1.25 & 55.85 & 59.76 & 68.62 \\
\hline 12 & 5.30 & 3.28 & 5.79 & 1.25 & 6.64 & 4.11 & 7.26 \\
\hline 13 & 1.96 & 1.41 & 1.66 & 1.21 & 2.38 & 1.72 & 2.02 \\
\hline 14 & 21.28 & 19.77 & 20.81 & 1.24 & 26.48 & 24.60 & 25.89 \\
\hline 15 & 11.68 & 7.51 & 12.75 & 1.25 & 14.57 & 9.37 & 15.91 \\
\hline Total & 258.40 & 207.83 & 221.12 & - & 322.76 & 257.07 & 274.79 \\
\hline Media & 17.23 & 13.86 & 14.74 & 1.27 & 21.52 & 17.14 & 18.32 \\
\hline
\end{tabular}

De acuerdo con el método topográfico el volumen medio de sedimento retenido por dique en Saldaña (Sñ) es $144.35 \mathrm{~m}^{3}$ y en Tórtoles (Tr) $17.23 \mathrm{~m}^{3}$; el total del sedimento retenido por todos los diques, asciende respectivamente a $3608.74 \mathrm{~m}^{3} \mathrm{y}$ $258.40 \mathrm{~m}^{3}$. Estos valores resultan un 4 a un 9\% superiores en Saldaña y un 17 a $24 \%$ en Tórtoles con respecto a los métodos geométricos. Al traspasar los volúmenes de sedimento a peso se obtienen $5691.97 \mathrm{t}$ (Sñ) y $322.76 \mathrm{t}$ (Tr). A partir de estos valores se ha calculado la tasa de sedimento media retenida $(S Y)$ por los diques en ambas zonas: $10.9 \mathrm{t} \mathrm{ha}^{-1}$ año ${ }^{-1}$ (Sñ) y $6.4 \mathrm{t} \mathrm{ha}^{-1} \mathrm{año}^{-1}(\mathrm{Tr})$, que son un 4 a un $14.5 \%$ mayores (Sñ) y un 19 a $26.7 \%$ (Tr) que con los métodos geométricos. Si atendemos a la eficacia de retención (TE), según el criterio de Brown and Jarvis (1943), el método topográfico asocia eficacias de retención entre un $97.7 \%$ (Sñ) y $94.4 \%$ (Tr), también superiores (Tr) o prácticamente iguales (Sñ) a las estimadas mediante los métodos geométricos. Esto conduce a que, con el método topográfico, se obtenga una emisión de sedimentos menor o igual a la de los métodos geométricos y que, por tanto, se deduzca un mayor rendimiento de los diques controlando la llegada de sedimento hacia los cauces próximos de la red de drenaje natural en ambas zonas (río Carrión y río Corneja, respectivamente).

Igualmente, al sumar $S Y$ con $S E$, obtenemos una mayor producción de sedimentos en las zonas estudiadas si trabajamos con el método topográfico: $11.02 \mathrm{t} \mathrm{ha}^{-1}$ año ${ }^{-1}$ y 6.9 $\mathrm{t} \mathrm{ha}^{-1} \mathrm{año}^{-1}(\mathrm{Tr})$, que suponen entre un 4.2-9.2 \% y un 18.3-25.6 \% (Tr) mayor. 
Tabla 3. Sedimentos retenidos (SY), eficiencia de retención de los diques (TE), emisión de sedimentos (SE); producción total de sedimentos o tasa de erosión (TSY), según los métodos: topográfico (A), prisma trapezoidal (B) y prisma piramidal (C), en Saldaña.

\begin{tabular}{|c|c|c|c|c|c|c|c|c|c|c|c|c|}
\hline $\begin{array}{l}\mathrm{N}^{\circ} \\
\text { diq. }\end{array}$ & $\begin{array}{c}\mathrm{SY}_{\mathrm{A}} \\
\mathrm{t} \cdot \mathrm{ha} \mathrm{a}^{-1} \mathrm{año}^{-1}\end{array}$ & $\begin{array}{c}\mathrm{SY}_{\mathrm{B}} \\
\text { thha } \\
\text { año }\end{array}$ & $\begin{array}{c}\mathrm{SY}_{\mathrm{C}} \\
\mathrm{t} \cdot \mathrm{ha^{-1 }} \mathrm{año}^{-1}\end{array}$ & $\begin{array}{l}\mathrm{TE}_{\mathrm{A}} \\
(\%)\end{array}$ & $\begin{array}{l}\mathrm{TE}_{\mathrm{B}} \\
(\%)\end{array}$ & $\begin{array}{l}\mathrm{TE}_{\mathrm{C}} \\
(\%)\end{array}$ & $\begin{array}{c}\mathrm{SE}_{\mathrm{A}} \\
\mathrm{t} \cdot \mathrm{ha}^{-1} \mathrm{año}^{-1}\end{array}$ & $\begin{array}{c}\mathrm{SE}_{\mathrm{B}} \\
\mathrm{t} \cdot \mathrm{ha} \mathrm{a}^{-1} \mathrm{año}^{-1}\end{array}$ & $\begin{array}{c}\mathrm{SE}_{\mathrm{C}} \\
\mathrm{t} \cdot \mathrm{ha} \mathrm{a}^{-1} \mathrm{año}^{-1}\end{array}$ & $\begin{array}{c}\mathrm{TSY}_{\mathrm{A}} \\
\mathrm{t} \cdot \mathrm{ha}^{-1} \mathrm{año}^{-1}\end{array}$ & $\begin{array}{c}\mathrm{TSY}_{\mathrm{B}} \\
\mathrm{t} \cdot \mathrm{ha}^{-1} \mathrm{año}^{-1}\end{array}$ & $\begin{array}{c}\mathrm{TSY}_{\mathrm{C}} \\
\mathrm{t} \cdot \mathrm{ha}^{-1} \mathrm{año}^{-1}\end{array}$ \\
\hline 1 & 42.72 & 18.41 & 21.83 & 99.78 & 99.50 & 99.58 & 0.09 & 0.09 & 0.09 & 42.82 & 18.50 & 21.92 \\
\hline 2 & 7.25 & 8.96 & 9.14 & 98.75 & 98.99 & 99.01 & 0.09 & 0.09 & 0.09 & 7.34 & 9.06 & 9.23 \\
\hline 3 & 9.43 & 5.64 & 4.65 & 98.95 & 98.26 & 97.90 & 0.10 & 0.10 & 0.10 & 9.53 & 5.74 & 4.75 \\
\hline 4 & 30.99 & 25.34 & 21.02 & 99.67 & 99.60 & 99.52 & 0.10 & 0.10 & 0.10 & 31.09 & 25.44 & 21.12 \\
\hline 5 & 2.46 & 1.31 & 1.09 & 96.29 & 93.28 & 92.02 & 0.09 & 0.09 & 0.09 & 2.55 & 1.41 & 1.19 \\
\hline 6 & 34.95 & 23.90 & 18.86 & 99.72 & 99.59 & 99.47 & 0.10 & 0.10 & 0.10 & 35.05 & 24.00 & 18.96 \\
\hline 7 & 7.98 & 9.04 & 10.40 & 98.77 & 98.91 & 99.05 & 0.10 & 0.10 & 0.10 & 8.08 & 9.14 & 10.50 \\
\hline 8 & 5.63 & 4.72 & 4.26 & 98.26 & 97.92 & 97.70 & 0.10 & 0.10 & 0.10 & 5.73 & 4.82 & 4.36 \\
\hline 9 & 7.42 & 9.28 & 10.27 & 98.77 & 99.01 & 99.11 & 0.09 & 0.09 & 0.09 & 7.51 & 9.37 & 10.36 \\
\hline 10 & 10.35 & 9.30 & 8.59 & 99.09 & 98.99 & 98.90 & 0.10 & 0.10 & 0.10 & 10.44 & 9.40 & 8.69 \\
\hline 11 & 9.10 & 8.05 & 7.74 & 98.93 & 98.80 & 98.75 & 0.10 & 0.10 & 0.10 & 9.19 & 8.15 & 7.84 \\
\hline 12 & 11.24 & 13.95 & 12.47 & 99.19 & 99.34 & 99.26 & 0.09 & 0.09 & 0.09 & 11.33 & 14.04 & 12.56 \\
\hline 13 & 13.01 & 21.94 & 15.03 & 99.24 & 99.55 & 99.34 & 0.10 & 0.10 & 0.10 & 13.11 & 22.04 & 15.13 \\
\hline 14 & 25.66 & 45.12 & 38.81 & 99.59 & 99.76 & 99.73 & 0.11 & 0.11 & 0.11 & 25.77 & 45.23 & 38.92 \\
\hline 15 & 5.90 & 6.88 & 6.81 & 98.33 & 98.56 & 98.55 & 0.10 & 0.10 & 0.10 & 6.00 & 6.98 & 6.91 \\
\hline 16 & 4.63 & 6.72 & 5.92 & 98.01 & 98.62 & 98.43 & 0.09 & 0.09 & 0.09 & 4.73 & 6.81 & 6.02 \\
\hline 17 & 6.78 & 8.12 & 7.77 & 98.58 & 98.81 & 98.76 & 0.10 & 0.10 & 0.10 & 6.88 & 8.22 & 7.86 \\
\hline 18 & 1.42 & 1.97 & 2.23 & 93.02 & 94.88 & 95.45 & 0.11 & 0.11 & 0.11 & 1.52 & 2.07 & 2.33 \\
\hline 19 & 1.37 & 2.47 & 2.20 & 93.30 & 96.19 & 95.73 & 0.10 & 0.10 & 0.10 & 1.46 & 2.57 & 2.29 \\
\hline 20 & 0.63 & 1.21 & 1.53 & 86.53 & 92.47 & 93.97 & 0.10 & 0.10 & 0.10 & 0.73 & 1.31 & 1.63 \\
\hline 21 & 10.39 & 12.36 & 9.76 & 99.14 & 99.28 & 99.08 & 0.09 & 0.09 & 0.09 & 10.48 & 12.45 & 9.85 \\
\hline 22 & 6.68 & 5.59 & 4.75 & 98.73 & 98.49 & 98.23 & 0.09 & 0.09 & 0.09 & 6.76 & 5.67 & 4.83 \\
\hline 23 & 3.87 & 2.78 & 2.62 & 97.68 & 96.79 & 96.61 & 0.09 & 0.09 & 0.09 & 3.96 & 2.87 & 2.71 \\
\hline 24 & 11.53 & 7.46 & 8.78 & 99.21 & 98.78 & 98.96 & 0.09 & 0.09 & 0.09 & 11.63 & 7.55 & 8.87 \\
\hline 25 & 1.71 & 1.64 & 1.80 & 94.97 & 94.78 & 95.21 & 0.09 & 0.09 & 0.09 & 1.80 & 1.73 & 1.89 \\
\hline Tot. & 273.11 & 262.2 & 238.32 & - & - & - & 2.41 & 2.41 & 2.41 & 275.52 & 264.6 & 240.72 \\
\hline $\mathrm{M}$ & 10.92 & 10.49 & 9.53 & 97.70 & 97.97 & 97.93 & 0.10 & 0.10 & 0.10 & 11.02 & 10.58 & 10.92 \\
\hline
\end{tabular}

Para ver si las diferencias detectadas son significativas se realizó un ANOVA y la prueba de Kruskal-Wallis, mediante el programa STATGRAPHICS Centurion XVI, con los resultados totales de producción de sedimentos (TSY) en ambas zonas. El ANOVA mostró para los dos casos que el p-valor fue superior a $0.05\left(p_{s \tilde{n}}=0.878\right.$ y $\left.\mathrm{p}_{\mathrm{Tr}}=0.924\right)$, lo que significa que no hay diferencias estadísticamente significativas con un nivel de confianza del 95\%. La Prueba de Kruskal-Wallis confirmó este resultado. No obstante, hay que considerar que estas diferencias, aunque no sean significativas, sí son importantes. Se ha visto que la diferencia de resultados entre el método topográfico y los geométricos oscilan desde un $4 \%$ a más de un $25 \%$. 
Tabla 4. Sedimentos retenidos (SY), eficiencia de retención de los diques (TE), emisión de sedimentos (SE); producción total de sedimentos o tasa de erosión (TSY), según los métodos: topográfico (A), prisma trapezoidal (B) y prisma piramidal (C), en Tórtoles.

\begin{tabular}{|c|c|c|c|c|c|c|c|c|c|c|c|c|}
\hline $\begin{array}{l}\mathrm{N}^{\circ} \\
\text { diq. }\end{array}$ & $\begin{array}{c}\mathrm{SY}_{\mathrm{A}} \\
\mathrm{t} \cdot \mathrm{ha}^{-1} \text { año-1 }\end{array}$ & 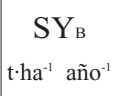 & $\underset{\text { th.ha-1 }}{S Y_{C}}$ & $\begin{array}{l}\mathrm{TE}_{\mathrm{A}} \\
(\%)\end{array}$ & $\begin{array}{l}\mathrm{TE}_{\text {в }} \\
(\%)\end{array}$ & $\begin{array}{l}\mathrm{TE}_{\mathrm{c}} \\
(\%)\end{array}$ & $\underset{t \text { tha }}{\mathrm{SE}_{\mathrm{A}}^{-1}} \mathrm{ann}$ & $\underset{t \cdot h \mathrm{a}^{-1}}{\mathrm{SE}_{\mathrm{B}}}$ & $\begin{array}{c}\mathrm{SE}_{\mathrm{c}} \\
\text { tha ha-1 } \\
\text { año- }\end{array}$ & $\begin{array}{l}\operatorname{TSY}_{\mathrm{A}} \\
\text { tha ha-1 } \text { año-1 }^{-1}\end{array}$ & $\begin{array}{r}\operatorname{TSY}_{\mathrm{B}} \\
\text { thata-1 año }\end{array}$ & $\begin{array}{c}\mathrm{TSY}_{\mathrm{C}} \\
\text { th.ha-1 año }\end{array}$ \\
\hline 1 & 1.42 & 1.16 & 1.07 & 95.42 & 92.42 & 91.81 & 0.07 & 0.10 & 0.10 & 1.48 & 1.26 & 1.16 \\
\hline 2 & 8.79 & 4.82 & 5.24 & 99.04 & 97.86 & 98.03 & 0.09 & 0.11 & 0.11 & 8.88 & 4.92 & 5.35 \\
\hline 3 & 2.35 & 1.91 & 2.10 & 97.82 & 95.48 & 95.87 & 0.05 & .09 & 0.09 & 2.40 & 2.00 & 2.19 \\
\hline 4 & 1.28 & 0.83 & 0.70 & 91.02 & 86.79 & 84.75 & 0.13 & 0.13 & 0.13 & 1.40 & 0.95 & 0.82 \\
\hline 5 & 3.09 & 2.58 & 2.35 & 96.59 & 95.40 & 94.98 & 0.11 & 0.12 & 0.12 & 3.20 & 2.70 & 2.48 \\
\hline 6 & 3.38 & 1.85 & 1.88 & 97.97 & 94.78 & 94.88 & 0.07 & .10 & .10 & .45 & 1.95 & 1.98 \\
\hline 7 & 8.35 & 9.04 & 7.52 & 99.33 & 99.13 & 98.96 & 0.06 & 0.08 & 0.08 & 8.40 & 9.11 & 7.60 \\
\hline 8 & 2.07 & 1.28 & 1.61 & 96.91 & 93.25 & 94.54 & 0.07 & 0.09 & 0.09 & 2.14 & 1.38 & 1.71 \\
\hline 9 & 38.47 & 24.63 & 25.99 & 99.83 & 99.62 & 99.64 & 0.07 & 0.09 & 0.09 & 38.53 & 24.72 & 26.08 \\
\hline 10 & 0.65 & 0.39 & 0.67 & 82.19 & 73.42 & 82.56 & 0.14 & 0.14 & 0.14 & 0.79 & 0.53 & 0.81 \\
\hline 11 & 23.72 & 25.38 & 29.14 & 99.60 & 99.57 & 99.62 & 0.09 & 0.11 & 0.11 & 23.81 & 25.49 & 29.25 \\
\hline 12 & 0.32 & 0.20 & 0.35 & 90.92 & 79.00 & 86.91 & 0.03 & 0.05 & 0.05 & 0.35 & 0.25 & 0.40 \\
\hline 13 & 0.42 & 0.30 & 0.36 & 87.12 & 76.69 & 79.45 & 0.06 & 0.09 & 0.09 & 0.48 & 0.40 & 0.45 \\
\hline 14 & 1.19 & 1.11 & 1.16 & 96.10 & 93.71 & 94.00 & 0.05 & 0.07 & 0.07 & 1.24 & 1.18 & 1.24 \\
\hline 15 & 0.45 & 0.29 & 0.49 & 85.93 & 75.65 & 84.06 & 0.07 & 0.09 & 0.09 & 0.53 & 0.39 & 0.59 \\
\hline Tot. & 95.95 & 75.77 & 80.63 & - & - & - & 1.16 & 1.46 & 1.46 & 97.1 & 77.2 & 82.1 \\
\hline Med. & 6.40 & 5.05 & 5.38 & 94.39 & 90.18 & 92.00 & 0.08 & 0.10 & 0.10 & 6.47 & 5.15 & 5.47 \\
\hline
\end{tabular}

Además si se observan los volúmenes obtenidos por los diques con cada método (Tab. 1 y 2) se observa que el volumen calculado con los métodos geométricos a veces es mayor que con el topográfico y otras veces es menor, lo cual produce una compensación de errores. Si se tienen en cuenta las diferencias absolutas (Fig. 2) la diferencia es realmente significativa ya que oscila entre el $26-29 \%$ (Sñ) y el $24 \%$ (Tr).

Por otro lado, hay que resaltar que los volúmenes calculados en este trabajo mediante los métodos geométricos están mejorados respecto a su utilización en otros trabajos. Esto se debe a que todas las variables empleadas en este estudio (longitudes, pendientes, perímetros de cuñas) han sido determinadas mediante estación total, mientras que en otros trabajos se obtienen con cinta métrica y GPS métricos, cuya precisión es muy inferior a la aquí presentada. Es muy probable entonces que si la comparación del método topográfico mediante estación total se hiciera con los métodos geométricos y sus medios asociados, las diferencias serían aún mayores. Por último hay que tener en cuenta que los métodos geométricos no tienen en cuenta diferencias altimétricas en la superficie de las cuñas de sedimentos, lo cual se traduce en una aproximación menos exacta de los volúmenes de las cuñas de sedimentos. También debe considerarse que los métodos geométricos deben de trabajar con lon- 


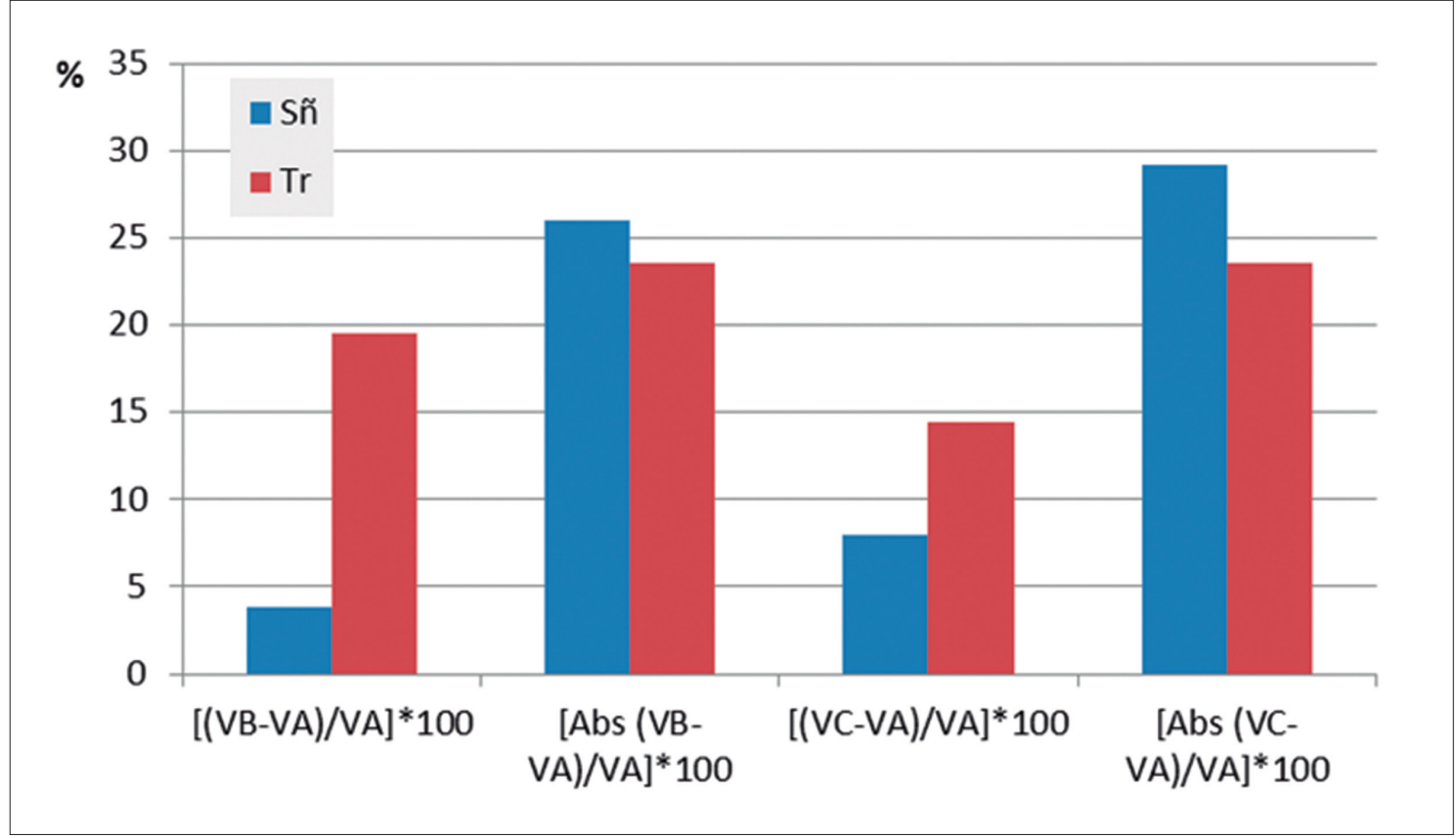

Figura 2. \% de las diferencias netas y absolutas (Abs) del volumen retenido por los diques en Saldaña (Sñ) y Tórtoles $(\mathrm{Tr})$ de los métodos geométricos $(\mathrm{B}, \mathrm{C})$ con respecto al método topográfico $(\mathrm{A})$.

gitudes de cuña corregidas según la pendiente de la cuña y esto no parece considerarse en los trabajos consultados (Castillo et al., 2007; Romero et al., 2007).

\section{Conclusiones}

De acuerdo con los resultados obtenidos en este trabajo se puede concluir que la estimación del sedimento retenido por los diques forestales, así como la determinación a partir del mismo de las tasas de producción de sedimentos en sus cuencas y de la emisión de sedimentos a la red de drenaje, varía en función de la metodología seguida. Dado que el método topográfico se basa en una representación detallada de las cuñas de sedimentos y en un cálculo más exhaustivo de los volúmenes, este método ofrece una mejor aproximación al papel que realizan los diques en el control de sedimentos en sus cuencas.

Los métodos geométricos, si bien son más sencillos y requieren un menor esfuerzo en campo, debido a que la amplitud de las diferencias puede superar más del $20 \%$, resultan recomendables sólo para realizar aproximaciones, pero no para realizar una evaluación exhaustiva de estas obras. Cualquier intento en este sentido debe fundamentarse en un conocimiento lo más exacto posible de los sedimentos retenidos.

La evaluación de diferentes tipos de hidrotecnias, especialmente los diques forestales, debe realizarse desde una suficiente aproximación a la realidad de sus efectos morfológicos, hidrológicos y sedimentarios, así como desde el análisis del ade- 
cuado diseño, emplazamiento y ejecución del cuerpo de obra y de las obras complementarias necesarias para su correcto funcionamiento. El diagnóstico sobre su eficacia nunca puede basarse en una aproximación parcial, no suficientemente detallada y sin considerar los criterios ingenieriles que inciden en su funcionamiento.

\section{Bibliografía}

Bellín N., Vanacker, V., van Wesemael, B., Solé-Benet, A., Bakker, M.M., 2011. Natural and anthopogenic controls on soil erosion in the Internal Betic Cordillera (southeast Spain). Catena, 87: 190-200. DOI: 10.1016/j.catena.2011.05.022

Boix-Fayos, C., Barberá G.G., López-Bermúdez, F., Castillo, V.M. 2007. Effects of check dams, reforestation and land-use changes on river channel morphology: Case study of the Rogativa catchment (Murcia, Spain). Geomorph., 91: 103-123. DOI: 10.1016/j.geomorph.2007.02.003

Boix-Fayos C, de Vente J, Martínez-Mena M, Barberá GG, Castillo V. 2008. The impact of land use change and check-dams on catchment sediment yield. Hydrol. Process., 22: 4922-4935. DOI: 10.1002/hyp.7115

Brown, C.B., Jarvis C.S. 1943. Discussion of Sedimentation in reservoirs, by J. Witzig. Proc. Amer. Soc. Civ. Eng., 69: 1493-1500.

Bryan, R.B., Yair, A. 1982. Badland geomorphology and piping. GeoBooks, Norwich.

Castillo, V.M., Mosch, W.M., Conesa, C., Barberá, G.G., Navarro, J.A., López-Bermúdez, F. 2007. Effectiveness and geomorphological impacts of check dams for soil erosion control in a semiarid Mediterranean Catchment: El Cárcavo (Murcia, Spain). Catena, 70: 416-427. DOI: 10.1016/j.catena.2006.11.009

Castillo, V., Boix, C., Vente, J. de, Martínez, M. y Barberá, G. 2011. Efectos del aumento de la cubierta vegetal y los diques de corrección en la producción de sedimentos de cuencas mediterráneas. Actas de la II Reunión sobre Hidrología Forestal. Cuad. Soc. Esp. Cienc. For., 32: 153-158

Clotet, N., Gallart, F., Sala, M., 1987. Los badlands, características, interés teórico, dinámica y tasas de erosión. Notes de Geogr. Fís., 15-16: 28-37

Díaz, V., Mongil, J., Navarro, J., 2014a. Propuesta de una nueva metodología para determinar la efectividad de los diques en la retención de sedimentos. Cuad. Inv. Geogr., 40 (1): 169-190.

Díaz, V., Mongil, J., Navarro, J., 2014b. Topographical evaluation of sediment retention by check dams in the Mediterranean Tórtoles badlands-restoration site (Ávila, Spain). $J$. Soils Sediments, 14: 2045-2056. DOI:10.1007/s11368-014-0958-5

Gallart, F., Marignani, M., Pérez-Gallego, N., Santi, E., Maccherini, S., 2013. Thirty years of Studies on badlands, from physical to vegetational approaches. A succinct review. Catena, 106: 4-11. DOI:10.1016/j.catena.2012.02.008

García-Ruiz J.M., Nadal-Romero E., Lana-Renault N., Beguería S. 2013. Erosion in Mediterranean landscapes: Changes and future challenges. Geomorph., 198: 20-36. DOI: 10.1016/j.geomorph.2013.05.023

IGME. 1982. Mapa Geológico de España. Escala 1.50000. Hoja 164 (Saldaña). Inst. Geol. Min. Esp. Minist. Industr. Dir. G. Min. Madrid. 
IGME; 2008. Mapa Geológico de España. Escala 1:50.000. Hoja 529: Santa María del Berrocal. Inst. Geol. Min. de Esp. Minis. Ind. Dir. G. Min. Madrid

Martínez de Azagra, A., Fernández de Villarán, R., Seseña, A., Méndez, C., Díez, J.M., Navarro, J., Varela, J.M., 2002. Metodología para la inventariación de diques forestales gavionados. Aplicación en la provincia de Palencia. Cuad. Soc. Esp. Cien. For., 13: 171181.

Mather A. E., Stokes M., Griffiths J. S. 2002. Quaternary landscape evolution: a framework for understanding contemporary erosion, southeast Spain. Land Degr. \& Dev., 13 (2): 89109. DOI:10.1002/ldr.484

Nadal-Romero E., Torri D., Yair A. 2013. Updating the badlands experience. Catena, 106: 13. DOI.10.1016/j.catena.2012.07.009

Navarro J., De Araújo J.C., Mongil J. 2014. Assessment of 80 years of ancient badlands restoration in Saldaña, Spain. Earth Surf. Process. Landforms, 39: 1563-1575. DOI: 10.1002/esp.3541

Ramos, I., Navarro, J., Bartolomé, D., Díaz, V., Mongil, J., 2013. Control de sedimentos y erosión en la restauración hidrológico-forestal de Saldaña (Palencia). Actas del VI Congr. Forest. Esp. Vitoria. 12.

Rodríguez-Lloveras X., Rodríguez-Caballero E., Solé-Benet A., Benito G. 2014. Estimación de la producción de sedimento durante un evento de lluvia extraordinario mediante láser escáner terrestre (TLS), en Schnabel, S. y Gómez Gutiérrez A. (Eds.) Avances de la Geomorfología en España 2012-2014. XIII Reunión Nacional de Geomorfología, Cáceres. ISBN: 978-84-617-1123-9: 343-346

Romero, A., Alonso, F., Martínez-Lloris, M., 2007. Erosion rates obtained from checkdam sedimentation (SE Spain). A multi-method comparison. Catena, 71: 172-178. DOI: 10.1016/j.catena.2006.05.011

Romero, A., Ruiz, J.D., Belmonte, F. 2011. Tasas de erosión hídrica en la región de Murcia. Bol. Asoc. de Geógr. Esp., 56: 129-153.

Romero, A., Marín, P., Ortiz, R., 2012. Loss of soil fertility estimated from sediment trapped in check dams. South-eastern Spain. Catena, 99: 42-53. DOI: 10.1016/j.catena.2012.07.006

Sougnez, N., van Wesemael, B., Vanacker, V. 2011. Low erosion rates measured for steep, sparsely vegeted catchments in southeast Spain. Catena, 84: 1-11. DOI:10.1016/j.catena.2010.08.010

USDA. 2010. Keys to Soil Taxonomy. http://soils.usda.gov/technical/classification (Consulta: 2-9-2012)

Verstraeten, G., Poesen, J. 1998. Flooding of properties and sedimentation in retention ponds in central Belgium. Modelling soil erosion, sediment transport and closely related hydrological processes. Proc. of a Symp. at Vienna. IAHS Publication, 249. IAHS Press, Wallingford. 187-193. 